HANNA JENNI

\title{
Die pronominalen Erweiterungen beim Imperativ und der Ausdruck verbaler Reflexivität im Ägyptischen
}

1. Der Imperativ und seine Verstärkungen

2. Imperativ $+r=k$

3. Imperativ $+n=k$

4. Imperativ + tw

5. Die komplementäre Distribution von $n=k$ und $t w$ - ein ergativisches Relikt

6. Zur Reflexivität und zum Gebrauch der pronominalen Erweiterungen beim Imperativ

6.1 Zum Gebrauch des Imperativs mit $t w$ und $n=k$

6.2 Explizite versus implizite verbale Reflexivität

6.3 Übersetzungsstrategien beim Imperativ mit $\underline{t} w$ und $n=k$

6.4 Zur Reflexivität und zum Gebrauch von $r=k$ und $(j) r=f$ beim Imperativ

7. Zur Frage der Kompatibilität von $r=k /(j) r=f$ und $t w / n=k$

8. Unerweiterter negierter Imperativ

\section{Der Imperativ und seine Verstärkungen}

Der häufigste Gebrauch des Imperativs, einer deontischen Modalform, die nur von der 2. Person gebildet wird, ist der unmarkierte. Der Ausdruck des pronominalen Subjekts („du“, „ihr") ist implizit, d.h. in der Oberfläche getilgt. Im Falle des Plurals ist dieser meist nicht markiert oder aber durch die Endung - $w$ oder durch Pluralstriche (für -.w). Der bloße Imperativ drückt einen pragmatisch neutralen Befehl aus, den ein Sprecher an einen Adressaten richtet: „Tu (es)!“.

Das Ägyptische hat die Möglichkeit, den Imperativ insofern zu ,,verstärken“, als das Subjekt explizit genannt wird": „Tu (es), du!“. Dies kann auf drei verschiedene Arten realisiert werden: durch Zufügung des enklitischen Pronomens der 2. Person, durch die Präposition $n+$ Suffix-

1 Alan Gardiner, Egyptian Grammar, London 31957, 258f., $\$ 337$, 1f.; Michel Malaise/Jean Winand, Grammaire raisonnée de l'égyptien classique (AegLeod 6), Liège 1999, 512 f., \$ 839. pronomen der 2. Person oder durch die Präposition $r+$ Suffixpronomen der 2. Person. Im folgenden sollen die Unterschiede zwischen den einzelnen Realisationen aufgezeigt werden. Sie sind sowohl syntaktisch wie auch pragmatisch bedingt.

\section{Imperativ $+r=k$}

Beispiele von Imperativen, die mit der Präposition $r$ plus Suffixpronomen der 2. Person verstärkt sind, kommen sowohl mit als auch ohne direktes Objekt vor:

$1 \mathrm{Zu}$ Beginn der Lebenslehre des Ptahhotep bittet der König den Wesir Ptahhotep: „Lehre ihn ( $s b 3 r=k$ ) zuerst (zu reden) Rhetorik! “ ${ }^{2}$

2 Die erste Maxime der Lehre des Ptahhotep beginnt folgendermaßen: „Spiel dich nicht auf wegen deines Wissens, (sondern) frage nach Rat ( $n \underline{d} \underline{\underline{d}} \underline{\underline{d}} r=k$ ) beim Unwissenden wie auch beim Wissenden! ${ }^{3}$

3 Ein Ratschlag Ptahhoteps lautet: „Pass auf ( $s 3 w \underline{t} w$, vgl. unten Beispiel 31), dass ein Verständiger nicht sage: ,Hör zu $(s \underline{d} m r=k)$ ! Wenn du wünschst $[. . .]^{\text {s. }}$ "

4 Spruch 148 der Sargtexte fordert auf: „Betrachtet (m33(.w) jr=tn) Horus, (ihr) Götter! ${ }^{15}$

5 Im Spruch 316 der Sargtexte heißt es: „Seht mich an (m33(.w) wj $r=\underline{t} n)$, Menschen und Götter! ‘6 $^{6}$

\footnotetext{
${ }^{2}$ Ptahhotep 37.

${ }^{3}$ Ptahhotep 52; 54 (P).

${ }^{4}$ Ptahhotep 612f.

${ }^{5}$ CT II, 222d.

${ }^{6} \mathrm{CT}$ IV, $100 \mathrm{~b}$.
} 
6 Re sagt im Spruch 157 der Sargtexte über Horus: „Legt ihn ( $d y \quad r=t n s w)$ auf sein Bett! ${ }^{* 7}$

7 Ein Dämon wird im Spruch 115 der Sargtexte zu Hilfe gerufen: „Komm ( $m j \boldsymbol{r}=\boldsymbol{k}$ $r=k$ ), Gewalttätiger! ${ }^{\prime{ }^{8}}$ In diesem Fall ist $r=k$ verdoppelt.

8 Ein Dialog in den Sargtexten lautet: „Iss! (wnm jr $=k$ ), sagen sie zu mir. - Ich will nicht für euch essen - Warum?, fragen sie mich. “"

Ohne auf die Verwendung von $r=k$ in anderen Satzarten einzugehen ${ }^{10}$, ist, was die Pragmatik angeht, zu vermuten, dass der mit $r$ verstärkte Imperativ die kommunikative Relation von Sprecher und Adressat im Blick hat. Der den Befehl erteilende Sprecher mahnt den den Befehl empfangenden Adressaten (das Subjekt des Imperativs) zur Akzeptanz des Befehls, wenn er damit rechnen muss, dass der Adressat quasi (noch) nicht auf Akzeptanz "geschaltet “ ist $^{11}$. Dabei ist nicht nur die Rolle des Adressaten, sondern auch diejenige des Sprechers aufgewertet, und man könnte umschreiben: „Iss, ich bitte dich“, „Komm, ich flehe dich an" (siehe auch 6.4). Deswegen stehen mit $r=$ verstärkte Imperative gerne am Anfang einer Sequenz von Aufforderungen. So verwendet in der Lehre Ptahhoteps nur die erste Maxime (Beispiel 2) diese Form. Der Sprecher sucht den Adressaten zu überzeugen, den Befehl, die Aufforderung oder die Bitte anzunehmen, d.h. er fordert ihn auf, ermuntert oder bittet ihn, sei es nachdrücklich oder höflich, der im Imperativ ausgedrückten Tätigkeit Folge zu leisten. Es fällt auf, dass $r=k$ im religiösen Spruchgut besonders häufig anzutreffen ist (vgl. Anm. 10). Die Verwendung dieser Form wird begünstigt durch die Funktion

\footnotetext{
${ }^{7}$ CT II, 342a.

${ }^{8}$ CT II, 134h.

${ }^{9}$ CT III, $48 \mathrm{e}-\mathrm{g}$.

${ }^{10}$ Dazu Ariel Shisha-Halevy, (l)rf in the Coffin Texts: A Functional Tableau, in: JAOS 106, 1986, 641658.

"Dem entspricht die Verwendung von $r$ bei den Verba dicendi; vgl. H. Jenni, „Sagen zu" im Ägyptischen, in: LingAeg 10, 2002, 239-259.
}

des magischen Textes als (zwingende) Beschwörung.

Häufig eignet sich als Übersetzung deutsches „bitte“ oder eine sogenannte Abtönungsparti$\mathrm{kel}^{12}$ wie „doch“; nicht selten ist aber $\varnothing$ vorzuziehen.

Dieselbe Funktion hat auch $m j$, ein semantisch erstarrter Imperativ der Bedeutung „komm!“, der einem Imperativ beigefügt werden kann ${ }^{13}$. Es handelt sich um den Aufruf der Distanzverringerung zwischen Befehlsempfänger und Befehlssender, d. h. um ein lokales Modell, das dem imperativischen Kommunikationsakt zugtundegelegt wird.

Eine andere Funktion als $r=k, r=t, r=\underline{t}$ hat die mit dem Suffixpronomen der 3. Singular $\mathrm{m}$. gebildete Partikel $(j) r=f$, die beim Imperativ eher selten zu sein scheint ${ }^{14}$. Siehe unten Beispiel 25 sowie:

9 Spruch 414 der Sargtexte: „Steig ein ( $h 3(j)$ $r=f$ in die nfrw. $t$-Barke! ${ }^{615}$

Das Pronomen der 3. Person kann sich nur auf den im Imperativ formulierten Verbalinhalt beziehen: „Steig ein, in Bezug auf es(, das Einsteigen)!“, im Sinne von: „Einsteigen ist es, was du zu tun hast". ${ }^{16}$

Auch für diese Verstärkung des Imperativs eignet sich als Übersetzung die Zufügung einer Abtönungspartikel wie „doch“, „schon“, „nur“, ,jetzt" etc.

${ }^{12}$ Zum Begriff vgl. Elke Hentschel/Harald Weydt, Handbuch der deutschen Grammatik, Berlin $1990,280-289$.

${ }^{13}$ Gardiner, Egyptian Grammar (wie Anm. 1), 259, $\$ 337,4$; Malaise/Winand, Grammaire raisonnée (wie Anm. 1), 513, \$ 839, ex. 1362.

${ }^{14}$ Gardiner, Egyptian Grammar (wie Anm. 1), 186, $\int 252,3 ; 258, \int 337,3$; Malaise /Winand, Grammaire raisonnée (wie Anm. 1), 513, \$ 839, ex. 1361.

${ }^{15} \mathrm{CT} \mathrm{V}, 247 \mathrm{~d}$.

${ }^{10} \mathrm{Ob} r=k$ sich zu $r=f$ entwickelt hat, wie zuweilen zu lesen ist, bleibt fraglich: Hellmut Brunner, Abriss der mittelägyptischen Grammatik, Graz ${ }^{2} 1967,26, \S 40$, c; Wolfgang Schenkel, Tübinger Einführung in die klassisch-ägyptische Sprache und Schrift, Tübingen 2005, 255. 


\section{Imperativ $+n=k$}

Nach der Besprechung des Imperativs mit $r=k$ und $(j) r=f$ und ersten Bemerkungen zu deren Pragmatik (vgl. auch 6.4) folgen nun zunächst einige Beispiele von Imperativen mit der Präposition $n$ mit Suffixpronomen der 2. Person:

10 Im Papyrus Westcar erhält König Snofru den Rat: „Rüste dir eine Barke aus ( $p r \quad n=k$ b3w) mit jeglichen schönen (Frauen) des Palastes! s $^{17}$

11 Die Lehre des Ptahhotep besagt: „Wenn du in leitender Stellung bist und dabei für zahlreiche Leute Befehle ausgibst, (dann) trachte nach jeglichem trefflichen Mittel $(h h(j) n=k s p n b \text { mnh }) !^{c / 18}$

12 Und an anderer Stelle: „[...], (dann) beschaffe dir Vertrauensleute $(j r j n=k$ mrw $n \boldsymbol{p h}$-jb)! (“19 $^{19}$

13 In der Lehre des Djedefhor heißt es: „Such $\operatorname{dir}<$ einen Verwalter> $(h h(j) n=k$ $<$ hnky>)! ‘20 $^{20}$

14 Im selben Text heißt es an anderer Stelle: „Wähle $\operatorname{dir}(s t p n=k$ hnr.t [...]) eine Strafanstalt $[\ldots] !^{c^{21}}$

15 Und Sinuhe wird gemahnt: „Denk an den Tag des Begräbnisses ( $s h \underline{3} n=k h r w n$ qrs)! $!^{c^{22}}$

16 In der Erzählung des Wenamun findet

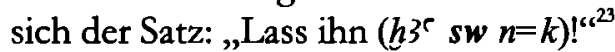

17 Die Lehre des Djedefhor gibt den Rat: „Nimm dir eine Frau $(j r(j) n=k$ hm.t) $[\ldots] !^{\varsigma^{24}}$

\footnotetext{
${ }^{17}$ Pap. Westcar 5, 2 f.

${ }^{10}$ Ptahhotep 86.

${ }^{19}$ Ptahhotep 233.

${ }^{20}$ Wolfgang Helck, Die Lehre des Djedefhor und die Lehre eines Vater an seinen Sohn, Wiesbaden 1984, 9 (und 11 zum Textproblem).

${ }^{21}$ Helck, Lehre des Djedefhor (wie Anm. 20), 19, DeM 1396; G 62 schreibt nur stp. Vgl. zur Stelle Alessandro Roccati, Su un passo di Hardjedef, in: JEA $68,1982,16-19$.

${ }^{22}$ Sinuhe B 190 .

${ }^{23}$ Wenamun 2, 47.

${ }^{24}$ Helck, Lehre des Djedefhor (wie Anm. 20), 4.
}

18 In einem Brief an einen nubischen Fürsten heißt es: $s h 3 n=k p 3 h r w n ~ m s ~ p 3 j n w$, „Denk an den Tag des Bringens des Tributs! (65 $^{25}$

19 Die Lehre des Ani besagt: „Leg dir einen Garten an $(j r(j) n=k h<s>b)$, umzäune dir ein Gurkenbeet (jnh $n=k \quad b(n) d . t)[\ldots]$, pllanze dir eine Sykomore $(d g 3 j n=k$ nh.t $)$ darin! c.6 $^{26}$

20 An anderer Stelle sagt Ani: $w h 3 n n=k g r$, „Wähle dir das Schweigen! ‘蛹

21 Auf einer Stele heißt es: $r m y \quad n=j n h y \quad n=j$ $r m \underline{t} . w \quad n b(. w) \quad s h 3 \quad n=\underline{k}<n>\operatorname{th}(j) \quad m \check{s} d h$ [... ] , ,Weint um mich, klagt um mich, alle Menschen! Denkt daran, euch mit $\check{s} d h$ zu betrinken $[\ldots] !^{c^{2 B}}$

22 Sinuhe wird aufgefordert: $j r(j) n=k j w . t r$ Km.t, „Komm nach Ägypten! (“9 $^{29} \mathrm{Um}$ schreibung des Imperativs mit $j r j$ : „mach dir ein Kommen"; so auch:

23 Ein altägyptisches Beispiel: $j r(j) \quad n=k$ $n d r(w) . t$, ,Pack an!" (,Mach dir ein Anpacken! $)^{30}$.

Bei den mit der Präposition $n$ verstärkten Imperativen fällt bei einer Durchsicht der Beispiele auf, dass - im Gegensatz zu $r$ - alle Verben ein direktes Objekt (in der Transkription hervorgehoben) haben ${ }^{31}$.

\footnotetext{
${ }^{25}$ Pap. Berlin 3043 (Koller), 5, 1, zitiert nach DZA 29.470.010 (http://aaew.bbaw.de/dza/index.html).

${ }^{26}$ Lehre des Ani B, 19, 1-3. Joachim Friedrich Quack, Die Lehre des Ani. Ein neuägyptischer Weisheitstext in seinem kulturellen Umfeld (OBO 141), Freiburg (Schweiz)/Göttingen 1994, 103; $297 \mathrm{f}$.

${ }^{27}$ Lehre des Ani B, 22, 10.

${ }^{29}$ Leiden V. 15, zitiert nach DZA 29.470.820 (http://aaew.bbaw.de/dza/index.html).

${ }^{20}$ Sinuhe B 188.

${ }^{30}$ Zitiert nach Elmar Edel, Altägyptische Grammatik, Rom 1955, Bd. 1, 302, § 624.

${ }^{31}$ In Urk. IV, 1087, 4, ist subjunktivisches $s \underline{d} m=f \mathrm{zu}$ verstehen $(m 3 n=k)$ : „Dann sprach seine Majestät zu ihm: Du sollst (nun) zu der Wesirshalle ( $\left.r p^{3} h 3 \quad n \quad t 3 t j\right)$ blicken: Sei wachsam [...]!“. Die beiden jussivischen Formen stehen nicht auf derselben Ebene: Der König fordert den Wesir optativisch auf, sich nun gedanklich seinem Amtssitz zuzuwenden; dann folgt ein erster Ratschlag in imperativischer Form. - Gegenbeispiele wie CT I, 58c (B3Bo), mit pronominalem und nominalem Dativ und CT II, $10 \mathrm{~d}$ (B1Bo), mit unpassendem $n=\underline{t}$ finden sich jeweils nur in einzelnen Versionen und
} 


\section{Imperativ $+\underline{t} w$}

Bei Imperativen mit enklitischem Pronomen der 2. Person ist dagegen auffallend, dass kein direktes Objekt vorhanden ist ${ }^{32}$ :

24 Vor der Eroberung Megiddos gibt Thutmosis III. seiner Truppe folgende Anweisung: „Legt euch ganz tüchtig ins Zeug (mh.w tn jqr sp 2), meine siegreichen Krieger! c $^{33}$

25 In der Inschrift auf dem nördlichen Obelisken der Königin Hatschepsut in Karnak heißt es: „Hört nun her ( $s \underline{d m} . w$ j $r=f$ th)! Ich gab dafür Elektron von bester Qualität und $m a ß$ es in Scheffeln wie Korn. “34

26 Heqanacht sagt im Brief zu Mersu: „Was die Überschwemmung auf unserem Land betrifft - du bist es, der es bebaut - weh allen meinen Leuten und weh dir! Siehe, ich erachte dich als verantwortlich dafür. Sei sehr tüchtig beim Ackerbau, pass gut auf ( $h^{3}$ tw sp 2)! !6 35

27 Nach der Rückkehr Sinuhes und der Begrüßung sagt der König zu den Anwesenden: „Er soll sich nicht fürchten und nicht von Schrecken erfüllt sein. Er soll Freund sein unter den hohen Beamten

sind mit falscher Umsetzung von Genus oder Person respektive mit missverständlichen Vorlagen zu erklären. Andererseits ist in CT VII, 311a, in allen Versionen ein Passiv anzunehmen, vgl. R O. Faulkner, The Ancient Egyptian Coffin Texts, Bd. 3, Warminster 1978, 140, Anm. 4. - Umgekehrt illustriert den Sachverhalt das folgende Beispiel: $s n m h n=k m j b m r j$, „Bete für dich mit einem liebenden Herzen, [dessen Worte alle verborgen sind. Er wird für dich sorgen, er wird hören, was du gesagt hast, er wird dein Opfer annehmen]" (Lehre des Ani B 17, 2). Mit "er" ist "Gott" gemeint. In diesem Satz ohne direktes Objekt ist $n=k$ ein Dativ in der Funktion des Objekts zum Verb (,bitte zu deinen Gunsten mit einem liebenden Herzen").

${ }^{32}$ Bei Schenkel, Tübinger Einführung (wie Anm. 16), 228, wird vermerkt, dass die enklitischen Pronomina nach meist intransitiven Verben stehen; die Präposition $n$ wird unter den Verstärkungen des Imperativs nicht aufgeführt (nur $t w$ und $j r=$ ).

${ }^{33}$ Urk. IV 660, 5. - Vgl. unten 6.3 mit Anm. 55.

${ }^{34}$ Urk. IV, 367, 13-15.

${ }^{35}$ J. P. Allen, The Heqanakht Papyri, New York 2002, Taf. 8, Kol. $1 \mathrm{f}$. und in der Mitte des Hofstaates rangieren. Begebt euch zu dem Boudoir (wd $3 . w$ th $r$ ( $h n w t j d w 3 . t)$, um ihm seine Aufwartung zu machen! ( $^{36}$

28 In der Lehre des Ani wird der Rat gegeben: „Verhalte dich stets nach der Sitte

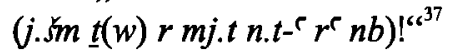

29 Ein Arbeiter spricht zum andern: $3 s \underline{t} w$, „Mach schnell!/Beeil dich!“ ${ }^{38}$

30 Am Ende der Erzählung des Oasenmannes sagt der König zu Rensi: „Triff selbst eine Entscheidung/entscheide du selber ( $w \underline{d}^{r} \underline{t} w \underline{d} s=k$ ), Sohn Merus! (c $39^{39}$

31 Eine Maxime in der Lebenslehre des Ptahhotep besagt: „Wenn du möchtest, dass deine Bedingungen optimal sind, so nimm dich in Acht vor jeglichem Bösen

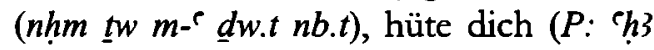
$\underline{t}(w)$, L1: $s 3(w) t(w)$, L2 mit Pseudopartizip: $s 33 . t j)$ vor (hr) der Habgier! ${ }^{40}$

32 Im Bericht über die Eroberung von Megiddo wird dem Heer befohlen: „Bereitet euch vor (grg.w th), bringt eure Waffen in Ordnung $\left(s s p d(. w) h^{\ulcorner} . w=\underline{t} n\right) !^{c^{41}}$

33 Im Spruch 892 der Sargtexte heißt es: „Verhülle $(h b s)$ dein Gesicht, verhülle dich ( $h b s \underline{t} w)$ mit $(m)$ seiner Haut ${ }^{142}$

34 In der Lehre des Djedefhor steht: ${ }^{b} \underline{t} w$ $h f t h r j r . t j=k j s 3 w t w$ c $b \underline{t w} k y$, (Reinige dich) Korrigiere dich vor deinen (eigenen) Augen, hüte dich, dass nicht ein anderer dich (reinige) korrigiere! (43 $^{43}$

35 Am Ende des ersten Spruches der Sargtexte heißt es: „O N.N., erhebe dich $(t s(j)$ tw) auf deine linke Seite, (gib dich) begib dich/dreh dich $(\underline{d}(j) \underline{t} w)$ auf deine rechte Seite! ! $^{44}$

${ }^{36}$ Sinuhe B 282

${ }^{37}$ Ani B, 19, 13.

${ }^{38}$ Urk. IV, 1142, 2.

${ }^{39}$ Oasenmann B 2, 133.

${ }^{40}$ Ptahhotep 299 f. Vgl. auch Ptahhotep 149, 281 und 612 (oben Beispiel 3).

${ }^{41}$ Urk. IV, 656, 1 f.

${ }^{42}$ CT VII, $103 \mathrm{a}-\mathrm{b}$ (Mü)

${ }^{43}$ Helck, Lehre des Djedefhor (wie Anm. 20), 4 CT I, 6b-c 
36 Im Buch von der Himmelskuh wird gewarnt: „Gib Acht wegen ('h3 tw hr) der Schlangen, die in dir sind [...] Bewache die (Schlangen), die in der Erde und im Wasser sind (s3w jmj.w $t 3 m w$ ) [...] Hütet euch, euer Spiel zu treiben (s3w.w $h^{\mathrm{c}} b$ (j)h.t nb.t) [...] Hüte dich ferner vor (s3w tw grt $r$ ) jenen Zauberern [...]! (45 $^{4}$

37 Ani rät: „Nimm (es) dir vor (jmj tw $r-h 3 . t=k)$ als einzuschlagenden Weg! [...] Nimm es dir vor (jmj sw $r-h 3 . t=k)$ unter deinen Geschäften! ${ }^{46}$

\section{Die komplementäre Distribution von $n=k$ und $\underline{t} w$ - ein ergativisches Relikt}

Offensichtlich verhalten sich also die sogenannten Verstärkungen mit enklitischem Pronomen und mit der Präposition $n$ syntaktisch komplementär zueinander, indem erstere ohne und letztere mit direktem Objekt vorkommen. Wie noch zu zeigen sein wird (6.1 und 6.4), stehen sie in pragmatischem Kontrast zu der Verstärkung mit der Präposition $r$.

Der Sachverhalt legt es nahe, in $n=$ eine Defektivschreibung von ursprünglichem $j n$ zur Markierung des Agens ${ }^{47}$ zu sehen und das komplementäre Paar $\underline{t} w / n=k$ mit dem dem Ägyptischen ursprünglich zugrundeliegenden (split-)ergativischen System zu erklären ${ }^{48}$. Das enklitische

\footnotetext{
${ }^{45}$ Erik Hornung, Der ägyptische Mythos von der Himmelskuh. Eine Ätiologie des Unvollkommenen (OBO 46), Freiburg (Schweiz)/Göttingen ${ }^{3} 1982$, 1820.

${ }^{46}$ Ani B 17, 13 und 15.

${ }^{47}$ So Pierre Grandet/Bernard Mathieu, Cours d'égyptien hiéroglyphique, Paris 1997, 271.

${ }^{48}$ Vgl. Massimo Patanè, Les traces de la construction ergative en égyptien, in: DE 10, 1988, 69-72; Pierre Grandet/Bernard Mathieu, La construction ergative de l'accompli égyptien, in: Sesto Congresso Internazionale di Egittologia. Atti, Bd. 2, Torino 1993, 145-151; Antonio Loprieno, Ancient Egyptian, Cambridge 1995, 65; Helmut Satzinger, On Ergativity in Egyptian, in: Andrzej Zaborski (Hg.), New Data and New Methods in Afroasiatic Linguistics, Wiesbaden 2001, 173-182 (mit weiterer Literatur); Antonio Loprieno, Egyptian Linguistics in the Year 2000, in: Zahi Hawass ( $\mathrm{Hg}$.), Egyptology at the
}

Pronomen $t w$, „du“, entspricht dem Absolutiv und steht bei Imperativen ohne direktes Objekt, (j) $n=k$, ,seitens deiner“, entspricht dagegen dem Ergativ und steht, wenn ein direktes Objekt vorhanden ist. Dabei ist jn phonetisch mit der Präposition $n$ zusammengefallen ${ }^{49}$.

Im nominativ-akkusativischen System hat sich die Distribution beim Imperativ auf der syntaktischen Ebene gehalten - „[...] imperatives typically preserve archaic grammatical patterns ${ }^{250}$-, wobei $t w$ akkusativische (z. B. 26 ,hüte dicb!"), $n=k$ dativische (z.B. 10 ,rüste dir'") Konnotation in reflexiver Konstruktion erhielten.

\section{Zur Reflexivität und zum Gebrauch der pronominalen Erweiterungen beim Imperativ}

\subsection{Zum Gebrauch des Imperativs mit $\underline{t} w$ und $n=k$}

Die Pronomina $t w$ und $n=k$ nach einem Imperativ dienten ursprünglich der Verstärkung oder Verdeutlichung des morphologisch

Dawn of the Twenty-first Century. Proceedings of the Eighth International Congress of Egyptologists, Cairo 2000, Cairo/New York 2003, Bd. 3, 79-84; ders., Ergativität und Diglossie: Die unsichtbare Hand in der ägyptischen Sprachgeschichte (Vortrag Berlin 2002, im Druck). - Ergativ-absolutivische Sprachen drücken (im Gegensatz zu den nominativ-akkusativischen Sprachen) das Subjekt eines intransitiven Verbs durch den passivischen unmarkierten Absolutus aus. Bei transitiven Verben steht dagegen das Subjekt einer Handlung, das in diesen Fällen dem Agens (Täter) entspricht, im Ergativ und das Patiens („Objekt") im Absolutus.

${ }^{49}$ Während das ergativische jn im akkusativischen System (außer in den sogenannten cleft sentences) durch $m$ - abgelöst wurde: Antonio Loprieno, Egyptian Linguistics in the Year 2000 (wie Anm. 48). - Auf die Frage von Pleneschreibungen der Präpsoition $n$ (Winfried Barta, Jn als Pleneschreibung der Präposition n, in: GM 103, 1988, 7-11; ders., Beispiele der Sargtexte für jn als Pleneschreibung der Präposition n, in: GM 197, 1989, 55-58; ders., Die Lexikostatistik der Pyramidentexte des Unas, in: GM 121, 1991, besonders 29f. ist hier nicht einzugehen.

So R. M. W. Dixon, Ergativity (Cambridge Studies in Linguistics, Bd. 69), Cambridge 1994, 201. 
schwach markierten Imperativs intransitiv respektive transitiv verwendeter Verben. Ihre syntaktische Distribution ist auf das ursprüngliche (split-)ergativische System des Äygptischen zurückzuführen und fungierte nach dem Übergang zum Kasus-System als Akkusativ respektive Dativ. Die Funktion der Pronomina blieb im Grunde dieselbe im Sinne einer "Langform“ des Imperativs durch pronominale „Verstärkung“, die aber besser als pronominale Erweiterung des Imperativs zu bezeichnen wäre, wobei die Erweiterung syntaktisch gesehen in der Zufügung einer reflexiven Konstituente besteht. Die Verwendung der pronominalen Erweiterung ist stark idiomatisch und prosodisch geprägt; überdies kann sie dazu dienen, kontextuelle Mißverständnisse auszuschalten.

Im Falle des Imperativs $s 3 w \underline{t w}$, „hüte dich!“, scheint es sich um einen Fall von Idiomatik zu handeln, im folgenden Beispiel vermutlich um einen von Prosodie und Variation:

38 In der Lehre des Ani heißt es: jmj $\underline{t}(w) r$ h3.t $=k r$ mj.t $n$ sm , „Setz, du, (es) vor dich (= Nimm es dir vor) als einzuschlagenden Weg“", und vier Verse weiter: jmj sw $r$-ḩ3.t $=k m n 3 y=k j p . w t$, ,Setz es vor dich (= Nimm es dir vor) unter deinen Geschäften! ‘ $^{51}$

\subsection{Explizite versus implizite verbale Reflexivität}

Die in diesem Zusammenhang interessante Frage, wann ein Verb ein Pronomen als reflexives direktes Objekt benötigt und wann nicht, ist für das Ägyptische bisher nicht untersucht worden.

Das Reflexivpronomen entfällt ganz beim Infinitiv ( $r 3 n$ sn $t 3 r \underline{d}(j) . t h \underline{h} \underline{h} . t$, „Spruch, die Erde zu küssen (und) sich auf den Bauch zu werfen ${ }^{*{ }^{52}}$ ) und beim Pseudopartizip. Das Fehlen des

\footnotetext{
${ }^{51}$ Ani B 17, 13-15.

${ }^{52}$ Z. B. DZA 26.260.490ff. (http://aaew.bbaw.de/ dza/index.html).
}

Reflexivpronomens beim Infiniv in der oben erwähnten Verwendung ist dadurch bedingt, dass er impersonal, d. h. agenslos verwendet ist. Beim Pseudopartizip ist es die durch die passive Diathese bedingte Agenslosigkeit (transitive Verben) respektive die durch die mediale Diathese bedingte Agensabwertung (intransitive Verben), die ein Reflexivpronomen ausschließt: Im Blick ist das Patiens, nicht das Agens. So heißt es beispielsweise $h r . k w j$, nicht ${ }^{*} h r . k w j w j$, für „ich habe mich vorbereitet/ich bin bereit", und bei $h 3 j . k w j$, ,ich bin hinabgegangen/ich befinde mich unten", geht es nicht darum, dass ich mich hinabbefördert habe, sondern dass ich unten bin. Das Agens ist abgewertet und mit dem allfälligen Nutznießer identisch (Medium). Dass also das ursprünglich absolutivische Akkusativpronomen beim medialen Pseudopartizip von Verben reflexiver Bedeutung nicht in Erscheinung tritt, leuchtet ein.

Ausgehend von dem zu den agenslosen und agensabwertenden Formen Gesagten müsste bestimmt werden können, in welchen Fällen agentivischer Verbalformen das reflexive direkte Objekt explizit genannt wird und in welchen nicht.

In Sätzen mit nichtabgewertetem Agens wird das ursprünglich absolutivische Pronomen zum direkten Objekt: Ergativisches „durch mich gewaschen ich" wird zu akkusativischem „ich wasche mich“. Dass das Pronomen der 3. Person nicht wie im Deutschen das reflexive und das nichtreflexive („sich“ vs. „ihn“) unterscheidet, erklärt sich ebenfalls dadurch. Sowohl $w^{c} b=f$ wie auch $w^{\mathrm{r}} b=f s w$ sind doppeldeutig. „er wäscht .../er wäscht sich“ respektive „er wäscht sich/er wäscht ihn". Wenn das Fehlen des Akkusativpronomens mit Agensabwertung zu tun hat, so würde $w^{c} b=f$ einem medialen Satz entsprechen, etwa „es wäscht sich an ihm/er wäscht-sich/er vollzieht Waschung an sich", während $w^{r} b=f s w$ - sofern der Kontext nicht eine andere Person meint - aktives ,er wäscht das Objekt sich selbst/er wäscht sich“, ohne Agensabwertung.

Mit der Agensabwertung verbunden ist Resultativität, so dass $w^{c} b=f$ das Resultat im Blick hat, während $w^{c} b=f s w$ einen (transitiven) Prozess ausdrückt. So findet sich das reflexive Pro- 
nomen auch bei der prozessualen pseudoverbalen Form $j w=f h r s d m^{33}$.

Der Imperativ ist eine agentivische Verbalform, weist jedoch im Ägyptischen eine schwache morphologische Kennzeichnung auf (siehe 1.), so dass er in seiner Pragmatik zur Verdeutlichung durch Zufügung des Subjektes tendiert. Die Beibehaltung der syntaktischen Distribution der ursprünglich ergativisch-absolutivischen Pronomina (5.) und die Tatsache, dass der Imperativ eo ipso resultativen Charakter hat, machen klar, dass im Falle von Imperativ $+\underline{t} w-$ im Gegensatz zu dem oben Gesagten - nicht ein Prozess ausgedrückt ist.

\section{3 Übersetzungsstrategien beim Imperativ mit $\underline{t} w$ und $n=k$}

Mit dieser Herleitung von $\underline{t} w$ respektive $n=k$ nach Imperativ wird verständlicher, $\mathrm{da} \beta$ sich Übersetzer in einem Dilemma befinden: Sollen sie $\underline{t} w$ respektive $n=k$ nach Imperativ als reflexives akkusativisches respektive dativisches Pronomen wiedergeben oder aber durch $\varnothing$ oder ein nominativisches Pronomen (z. B. „songe, toi ..." $\left.{ }^{54}\right)$ ? In der Regel entscheiden sie sich für die erste Lösung, sofern sie ein passendes Verb finden, das sich reflexiv verwenden lässt. Die Erklärung von $\underline{t} w$ und $n=k$ nach Imperativ als
Verstärkung desselben dürfte überhaupt auf diejenigen Fälle zurückgehen, wo die erstgenannte Lösung nicht praktikabel zu sein schien. Überspitzt gesagt: Unsere Grammatiken wissen vom sogenannten verstärkten Imperativ (von $r=k$ hier abgesehen, dazu unten 6.4) nur, weil die europäischen Sprachen bei intransitiver Konstruktion reflexive und nichtreflexive Verben lexikalisch unterscheiden, d.h. in der Oberflächenstruktur unterscheiden müssen.

$\mathrm{Da}$ die ägyptischen Verben - wie wenige Blicke in ein Wörterbuch zeigen - sowohl intransitiv, transitiv wie auch reflexiv gebraucht werden können (so z.B. $w^{c} b$, „rein sein/sich-reinigen/ reinigen“, nhm, „retten/sich-retten“ etc.), sind die syntaktischen Kategorien jedoch auf der Seite der Übersetzungssprache relevant, nicht auf derjenigen der Ausgangssprache. Die Scheidung in Fälle, wo „Verstärkung“, d. h. referenzidentisches nominativisches Pronomen, und andererseits in solche, wo reflexivisches akkusativisches respektive dativisches Pronomen vorliegen soll, ist jedoch willkürlich, weil sie von der Übersetzungssprache gesteuert ist. So werden häufig nur diejenigen Fälle von $t w$ respektive $n=k$ nach Imperativ als „eigentliche Verstärkung“" des Imperativs anerkannt, bei denen der Übersetzer keine adäquate reflexive Wendung zur Hand hat. Die gängige, sich in der Übersetzung zeigende Auffassung scheint etwa die zu sein:

\begin{tabular}{|c|c|c|c|}
\hline \multicolumn{2}{|c|}{ intransitiv } & \multicolumn{2}{|c|}{ transitiv } \\
\hline$m h . w \underline{t} n$ & $\underline{t} s j \underline{t} w$ & $\operatorname{sh} 3 n=k h r w n$ qrs & $j r j n=k h s b$ \\
\hline $\begin{array}{l}\text { A) Packt an, ihr! } \\
\text { nominativisch reflexiv: } \\
\text { angeblich betont }\end{array}$ & $\begin{array}{l}\text { B) Erhebe dich! } \\
\text { akkusativisch } \\
\text { reflexiv: } \\
\text { angeblich unbetont }\end{array}$ & $\begin{array}{c}\text { A) Denke, du, an den Tag des } \\
\text { Begräbnisses! } \\
\text { nominativisch reflexiv: } \\
\text { angeblich betont }\end{array}$ & $\begin{array}{l}\text { C) Leg dir einen Garten } \\
\text { an! } \\
\text { dativisch reflexiv: } \\
\text { angeblich unbetont }\end{array}$ \\
\hline $\begin{array}{l}\text { Aber akkusativisch reflexiv: } \\
\text { B) Legt euch ins Zeug! }\end{array}$ & & $\begin{array}{l}\text { Aber dativisch reflexiv: } \\
\text { C) Mach dir Gedanken über } \\
\text { den Tag des Begräbnisses }\end{array}$ & \\
\hline
\end{tabular}

\footnotetext{
${ }^{53}$ Pap. Chester Beatty VII, verso, 1, 5.

${ }^{54}$ Malaise/Winand, Grammaire raisonnée (wie Anm. 1), 513, ex. 1358.
}

\footnotetext{
${ }^{55}$ Zugrundeliegend ist „macht euch vollständig!“.
} 
Die Übersetzungsstrategie A ist impraktikabel und findet sich kaum außerhalb von Grammatiken, dort als Verdeutlichung; vorzuziehen ist $\varnothing$ für $\underline{t} w$ und $n=k$. Diese Übersetzung A macht nur Sinn, wenn der Adressat ein Teil mehrerer potentieller Adressaten ist und hervorgehoben werden soll (z.B. „Geht ibr schon voraus!“, „Komm $d u$ wenigstens! $\left.{ }^{\circ}\right)^{56}$. Bei den intransitiven Beispielen ist $\mathrm{B}$ die übliche Übersetzung, wenn sich im Deutschen eine reflexive Entsprechung findet, A hingegen, wenn eine entsprechende Übersetzung entweder nicht gesucht oder als semantisch oder stilistisch unpassend verworfen wird. C kommt häufig vor, da ein Dativus commodi (Dativ des Interesses, der Gefälligkeit), d.h. ein „freier Dativ" (nicht: Dativ als Objekt zum Verb) ${ }^{57}$ sozusagen in jedem transitiven Satz recht gut tönt. Wenn - innerhalb der mit pronominalen Erweiterungen versehenen Imperative - in betonte und unbetonte unterschieden werden könnte oder sollte, indem akkusativische und dativische reflexive Konstruktionen als unbetont ausgeschieden werden, dann müsste sich dies am Gebrauch des Ägyptischen mes$\operatorname{sen}^{58}$. (Zur Verwendung von pronominalen direkten Objekten in reflexiver Bedeutung siehe oben 6.2.)

Wie sehr das Problem von der Übersetzungssprache ${ }^{59}$ und von der Wahl des Verbs und sei-

${ }^{\text {s6 }}$ Gerhard Helbig/Joachim Buscha, Deutsche Grammatik, Berlin etc. 2001, 185.

57 Nach Helbig/Buscha, Deutsche Grammatik (wie Anm. 56), 189; 263; 463.

${ }^{58}$ Vgl. Kurt Sethe, Das aegyptische Verbum im Altaegyptischen, Neuaegyptischen und Koptischen, Bd. 2, Leipzig 1899, 205, am Beispiel von $s 3 w(. w) t n:$ „[...] doch könnte das Pronomen hier auch reflexives Objekt sein, da sich das Verbum $s 3 w$ sowohl mit intransitiver Bedeutung ,sich hüten' als mit transitiver hüten findet."

${ }^{59} \mathrm{Vgl}$. als französische und englische Übersetzungen von Beispiel 15 ( $s h 3 n=k h r w n$ qrs): „Pense au jour de l'enterrement" (Gustave Lefebvre, Romans et contes égyptiens de l'époque pharaonique, Paris 1949, 17); "Think of the day of burial" (Miriam Lichtheim, Ancient Egyptian Literature, Bd. 1, Berkeley etc. 1973, 229); ,and have in mind the day of burial" (R. B. Parkinson, The Tale of Sinuhe and other Ancient Egyptian Poems 1940-1640 BC, Oxford 1997, 36); und von Beispiel 27 (wd3.w $\underline{n} r$ r $h n w t j d w 3 . t$ ): „Allez donc vers l'appartement du matin" (Claire Lalouette, Textes sacrés et textes profanes de l'ancienne Égypte, Paris ner Valenz abhängt, sei an folgendem umgekehrten Beispiel gezeigt, wo ein reflexiver Dativ „vermisst“" wird:

39 Eine Dienerin sagt zu ihrer Herrin: jr(j) $h r w n f r,{ }^{60}$ was in der Übersetzung der Urkunden IV „wörtlich“" mit „Mache einen schönen Tag!" wiedergegeben wird.

Wählt man „machen“ als Verb in der Übersetzungssprache, so würde hier ein "dir" ausgezeichnet passen („Mach [dir] einen schönen Tag!'), ja wäre eigentlich geboten, da die Wendung nur mit diesem Zusatz als semantisch korrektes Deutsch gelten kann. Aber $n=\underline{t}$ steht nicht da. Die Alternative wäre „Feiere einen schönen Tag!“‘.

Die pronominale „Verstärkung“ mit $t w$ und $n=k$ äußert sich als Zufügung einer reflexiven Konstituente, wodurch der Satz eine Valenzerhöhung erfährt. Ob sich die Konstituente mit derjenigen der Übersetzungssprache deckt oder nicht, ist an sich irrelevant. Es fällt beim Übersetzen offenbar schwerer, ein Wort nicht wiederzugeben als eines zuzufügen, vgl. Beispiel 25 ( $s \underline{d m}$ jr=ft $\boldsymbol{n}$, „Höret doch, Ø!') mit Beispiel 39 (ir(j) Ø hrw nfr, „Mach dir einen schönen Tag!') und 18 (sh3 $n=k \emptyset_{p 3}$ hrw [...], ,denk an den Tag $\left.[\ldots . .] !^{*}\right)$.

\subsection{Zur Reflexivität und zum Gebrauch von $r=k$ und $(j) r=f$ beim Imperativ}

Die Präposition $r+$ Pronomen der 2. Person nach dem Imperativ transitiv wie auch intransitiv verwendeter Verben realisiert ebenfalls eine "Gewichtung" des Imperativs, aber in einem spezifischeren pragmatischen Sinn (siehe 2.). Die syntaktische Erweiterung entspricht hier einer Konstituente, die nicht einem dem Verb angegliederten semantisch-logischen Adjunkt entspricht, und das Pronomen der 2. Person ist nicht semantisch referenzidentisch, sondern nur

1987 , Bd. 2, 237); "Proceed to the robing-room" (Lichtheim, a. a. O., 232); aber ,Allez, vous, au pavillon du matin" (Lefebvre, a.a.O., 23), wozu das oben Gesagte (mit Anm. 56) zu vergleichen ist.

${ }^{60}$ Urk. IV, 1164, 2. 
formal referenzidentisch. Pragmatisch gesehen nimmt das Pronomen der 2. Person Bezug auf die außerhalb des Satzes liegende 1. Person, den Sprecher, der mit dem Adressaten kommuniziert. Daher entspricht ein Imperativ mit $r=k$ einer (höflichen oder eindringlichen) Bitte. Die Zufügung von $r=k$ ist ein Modalitätsverfahren, das im Deutschen vielfach durch eine sogenannte Abtönungpartikel ausgedrückt ist ${ }^{61}$.

Im Falle von $(j) r=f$ (siehe 2.) ist die Präpositionalphrase ebensowenig semantisch bedingt wie bei $r=k$. Die 3. Person rekurriert hier auf den Verbalinhalt und hebt diesen hervor. Die Modalität geht in Richtung Dringlichkeit („,hör schon!"), Ungeduld (,hör mal!') o. ä

\section{Zur Frage der Kompatibilität von $r=k /(j) r=f$ und $t w / n=k$}

Beispiele, wo die beiden Arten der Verstärkungen des Imperativs, d. h. mit $r=$ und mit $\underline{t} w$ oder $n=k$, zusammen vorkommen, sind sehr selten, vgl.

$40 \underline{t} s \underline{t} w r=k j t=j W s j r$, ,Erhebe dich doch, mein Vater Osiris! ${ }^{6{ }^{63}}$ und

$41 \not h w(j . w) \underline{t n} j r=\underline{t n}$ ' $q n . w h r$, ,Schützt euch doch, ....(?)-Gesichtige! ${ }^{66^{64}}$.

Beispiel 41 ist nicht stichhaltig, da der pluralische (aber ohne Pluralstriche geschriebene) Imperativ auch als optativisches $s \underline{d} m=f$ zu interpretieren sein könnte. Anhand dieses Beispiels hat sich Shisha-Halevy ${ }^{65}$ gegen die Kompatibilität von $r=$ und dem abhängigen Pronomen ausgesprochen, und die Seltenheit der aufzutreibenden Fälle scheint ihm recht zu geben.

\footnotetext{
${ }^{61}$ Vgl. Anm. 12.

${ }^{62}$ Der häufigste Gebrauch von $(j) r=f$ ist derjenige als ,interrogative dialogue constituent " (häufig wie englisches ... do you?): Shisha-Halevy, (l)rf in the Coffin Texts (wie Anm. 10), 650; David P. Silverman, Interrogative Constructions with $J N$ and $J N-J W$ in Old and Middle Egyptian, Malibu 1980, besonders $93 \mathrm{ff}$.

${ }^{63} \mathrm{CT} \mathrm{VI,} 217 \mathrm{~m}$.

${ }^{64} \mathrm{CT}$ I, $218 \mathrm{~b}$.

${ }^{65}$ Shisha-Halevy, $(I) r f$ in the Coffin Texts (wie Anm. 10), 645, Anm. 8.
}

Zweimalige explizite Nennung des Subjekts des Imperativs, also des Adressaten, scheint dann nicht als pleonastisch zu gelten, wenn $\underline{t} w$ für das Verständnis des Satzes notwendiges Komplement ist. Es ist kein Zufall, dass der Satz 40 (und auch 41, sofern als Imperativ zu verstehen) ohne tw (respektive $\underline{t} n$ ) missverständlich wäre, indem der folgende Vokativ als direktes Objekt aufgefasst werden könnte (40: „Erhebe doch meinen Vater Osiris!“, 41: Schützt doch die ...(?)-Gesichtigen!" $)^{66}$.

Es sei hier auch Beispiel $25(s d m . w j r=f \underline{t} n)$ betrachtet, um festzustellen, dass das personal nicht an die 2. Person angepasste $(j) r=f$ vor dem abhängigen Pronomen steht. Die Partikel $(j) r=f$ (vgl oben 2. und 6.4) hat hier wohl zusätzlich die Funktion, den mit $\underline{\underline{t}} \boldsymbol{n}$ verstärkten Imperativ als solchen herauszustellen, da ohne die Partikel vielleicht ein optativisches $s \underline{d m}=f$ oder $\underline{t} n$ als direktes Objekt missverstanden würde. Dieser Zusatz scheint bei pluralischen Imperativen mit tn häufig zu sein ${ }^{67}$.

Beispiele für $n=k r=k+$ direktes Objekt lassen sich aus den oben genannten Gründen des Pleonasmus vermutlich nicht beibringen.

\section{Unerweiterter negierter Imperativ}

Zum Schluss kann man sich die Frage nach dem negierten Imperativ stellen. Bei der negativen Entsprechung des Imperativs, die mit $m$, dem Imperativ des Verbs jmj, „nicht (tun)“ plus folgendem sogenanntem Negativ-Komplement gebildet wird, sind Verstärkungen nicht anzutreffen. Das hängt syntaktisch mit dem „Dilemma" zusammen, dass $\underline{t} w$ respektive $n=k$ als Subjekt respektive Komplement eigentlich nach $m$ stehen müsste, als reflexiver Akkusativ respektive Dativ aber sinngemäß zum Verb gehört

${ }^{\circ 6}$ Malaise/Winand, (wie Anm. 1), 513, ex. 1360 , bezeichnen $t w$ in unserem Beispiel 40 explizit als „un objet direct et non le moyen de renforcement de l'impératif".

Sethe, Verbum (wie Anm. 58), Bd. 2, 206; Gardiner, Egyptian Grammar (wie Anm. 1), 186, $\$ 252,3$. 
und nach dem Negativ-Komplement zu stehen hätte: ${ }^{68}$

a) ${ }^{*} m$ tw snd.w, ,nichte, du, dich zu fürchten“, versus b) ${ }^{*} m$ snd.w $t w$, ,nichte, zu fürchten dich".

a') ${ }^{*} m(j) n=k \quad$ pr.w b3w, „nichte, du, zu rüsten eine Barke“, versus b') ${ }^{*} m \quad$ pr.w $n=k$ $b 3 w$, „nichte, zu rüsten dir eine Barke“.

In diesen Sätzen ist unter a) das zu Vermutende nach ergativischem Muster, unter b) nach akkusativischem Muster aufgeführt. Die beiden mit b) bezeichneten Sätze sind jedoch völlig unmöglich, weil das Ägyptische bei agenslosen Formen (Infinitv, Negativ-Komplement) oder Formen mit Agensabwertung (Pseudopartizip) keine Reflexivpronomina verwendet (siehe 6.2).

Ein Argument im Sinne der linguistic universals, das die explizite Nennung des Subjekts ebenfalls als höchst unwahrscheinlich erscheinen lässt, ist die Semantik des negativen Imperativs: Sie blendet den Subjektnexus zugunsten des negativen Skopus aus ${ }^{69}$. Denkt man sich eine Skala von negativ formulierten Modalformen, so könnte sie mit einer Bitte beginnen und über ein Verbot zur verzweifelten interjektiven Warnung laufen, etwa: „Ich bitte dich, nicht mit den Fingern zu essen - Du sollst nicht mit den Fingern essen Iss nicht mit den Fingern - Nicht mit den Fingern essen! - Nein!“

Bei Verboten scheint auch $r=k$ nicht vorzukommen. In diesem Fall müsste $r=k$ logischerweise hinter $m$ stehen $\left({ }^{*} m r=k\right.$ snd. $w$, „nichte, ich bitte dich, zu fürchten!"), was aber wegen der Univerbierung von $m+$ Negativ-Komplement nicht möglich ist.

\section{RÉSUMÉ}

Le présent article étudie les différentes extensions pronominales subséquentes à l'impératif égyptien. L'addition du pron. dép. $2^{c}$ pers. ainsi que celle de la préposition $n+$ pron. suff. $2^{\mathrm{e}}$ pers. forment un groupe complémentaire: $n=k$ est utilisé avec et $\underline{t} w$ sans objet direct. Ce fait peut être expliqué comme

\footnotetext{
${ }^{68}$ Das Verb jmj ist im folgenden mit dem im Deutschen nichtexistenten Verb "nichten“ wiedergegeben, um der ägyptischen Konstruktion zu folgen.

${ }^{69}$ Dankenswerter Hinweis von Antonio Loprieno.
}

trace de la (semi-)ergativité originale de la langue égyptienne, si on accepte de voir en $n$ non la préposition, mais la particule $j n$, ancienne marque de l'ergatif (devenue marque de l'agent mis en relief dans la langue du temps historique), tw étant la marque de l'absolutif (cf. la proposition nominale $n f r$ sw). Comme les impératifs ont tendance à conserver les caractères grammaticaux archaïques, la distribution syntaxique fut retenue, mais dans le système accusatif de la langue historique $n=k$ et $\underline{t} w$ furent compris comme datif réflexif et comme accusatif réflexif. L'addition de $n=k$ et de $t w$ constitue un moyen de mise en relief ou plutôt d'explicitation du sujet de l'impératif morphologiquement faiblement marqué. Il n'y a aucune raison de distinguer les impératifs avec mise en relief du sujet de ceux sans mise en relief du sujet, parce qu'une telle distinction n'est contrôlée que par la valence du verbe choisi par le traducteur. L'extension de l'impératif avec $n=k$ et tw semble dépendre largement de l'idiome et de la prosodie.

L'addition de $r+$ pron. suff. $2^{\mathrm{c}}$ pers. après un impératif s'explique comme moyen pragmatique par lequel le locuteur peut exprimer envers l'adressé une demande polie, emphatique ou même pressante. Concernant $r+$ pron. suff. $3^{c}$ pers., $(j) r=f$, le pronom se réfère au verbe, paraphrasant „'c'est ... que tu dois faire". Les deux sortes d'additions avec $r$ correspondent en général aux particules de modalité des langues européennes et sont incompatibles avec $n=k$ et $\underline{t} w$; dans des cas exceptionnels $\underline{t} w$ peut toutefois être utilisé pour éviter des malentendus.

Des considérations générales sur l'utilisation des pronoms réflexifs mènent à la constatation que les formes passives et médiales, c'est-à-dire les formes sans agent ou avec un agent à agentivité réduite, n'utilisent jamais un pronom réflexif explicite. Les formes avec agent explicite, par contre, utilisent le pronom réflexif explicite quand le verbe exprime un procès (et non un résultat, auquel cas le pronon réflexif est non explicite). Parce que les impératifs sont a priori résultatifs, cette distinction ne fonctionne pas avec les impératifs. Les impératifs négatifs n'ont jamais une extension pronominale pour la raison que le complément négatif est une forme sans agent.

\section{SUMMARY}

In this article the different pronominal extensions of the egyptian imperative are studied. The addition of the encl. pron. 2nd pers. as well as the preposition $n+$ suff. pron. 2 nd pers. are to be seen in parallel, as they are used in syntactic complementation: $n=k$ with and $t w$ without direct object. This can be explained as a relic of the original (split-)ergativity of the egyptian language, accepting that $n$ is actually the ergative (in historical times stressed agentivity) marker $j n$, 
whereas tw corresponds to the absolutive (cf. the nominal sentence $n f r \quad s w$ ). Since imperatives are disposed to preserve archaic grammatical patterns, the syntactical distribution was kept, but in the case system of the historical language $n=k$ and $\underline{t} w$ were understood as a reflexive dative and as a reflexive accusative, respectively. The addition of $n=k$ and $t w$ is a device of stressing or rather elucidating the subject of the morphologically weakly marked imperative. There is no reason to divide into stressed and unstressed imperatives, as such a division is controlled merely by the valence of the verb used in the translators language. The extension of the imperatif with $n=k$ and $\underline{t} w$ seems to depend largely on idiomatics and prosody.

The addition of the preposition $r+$ suff. pron. 2nd pers. after an imperative is explained as a pragmatic device that enables the speaker to express a polite, emphatic or forcible demand to the addressee. As for the construction $r+$ suff. pron. 3rd pers. sing., $(j) r=f$, the pronoun refers to the verb, paraphrasing "... is it, what you must do". Both additions with $r$ generally correspond to modal particles in european languages and are incompatible with $n=k$ and $t w$, save exceptions with $t w$ serving as a means of avoiding misunderstandings.

General considerations about the use of reflexive pronouns show that passive and medium forms, i.e. forms without agent or with marginalized agent, never use the explicit reflexive pronoun, whereas forms with explicit agent use, reflexive pronouns when a process (rather than a result, in which case the reflexive pronoun is not explicit) is expressed. Since the imperative has by itself resultative meaning, this distinction does not apply to imperatives. Negative imperatives never have a pronominal addition for the reason that the negative complement is agentless. 